\title{
Nordiques
}

33 | 2017

La transition des villes nordiques : quelles innovations territoriales en périphérie?

\section{Le sens véritable de la formule <sakumukmini> sur l'inscription de Rök}

\section{Alain Marez}

\section{(2) OpenEdition}

\section{Journals}

Édition électronique

URL : https://journals.openedition.org/nordiques/3510

DOI : $10.4000 /$ nordiques.3510

ISSN : 2777-8479

Éditeur :

Association Norden, Bibliothèque de Caen la mer

Édition imprimée

Date de publication : 1 mai 2017

Pagination : 129-137

ISBN : 978-2-9544654-9-4

ISSN : $1761-7677$

\section{Référence électronique}

Alain Marez, «Le sens véritable de la formule <sakumukmini> sur l'inscription de Rök », Nordiques [En ligne], 33 | 2017, mis en ligne le 17 janvier 2022, consulté le 02 février 2022. URL : http:// journals.openedition.org/nordiques/3510 ; DOI : https://doi.org/10.4000/nordiques.3510 


\section{Le sens véritable de la formule $<$ sakumukmini> sur l'inscription de Rök}

Alain Marez ${ }^{*}$

\section{RÉSUMÉ}

Une formule structure de façon insistante le célèbre monument runique suédois de Rök <sakumukmini>, dont le déchiffrement et donc linterprétation et la traduction demeurent encore problématiques. Heureusement, le graveur a réécrit la séquence des runes scandinaves à 16 signes en runes germaniques (fupark à 24 signes), rendant ainsi le texte intelligible. Une analyse épigraphique rigoureuse et méthodique de l'orthographe runique mise en cuvre dans la série en runes anciennes permet seule de restituer la chaîne phonique sous-jacente : cette étude aboutit à des conclusions bien éloignées des interprétations proposées jusquiici et projette un éclairage nouveau sur la tâche que s'assigne le graveur: en inscrivant des runes sur toute la surface du bloc de pierre, il donne naissance à un monument sans pareil, le seul digne à ses yeux de célébrer la mémoire de son fils défunt.

\section{ABSTRACT}

On the famous Swedish Rök stone, the runic formula <sakumukmini> appears regularly: its deciphering, interpretation and translation still raise many questions. Fortunately, the engraver wrote the sequence both in Scandinavian runes (16 signs) and in Germanic runes (fupark with 24 signs), thus making the text intelligible. A rigorous epigraphic analysis of the runic spelling in the series of ancient runes allows to reconstruct the underlying phonic chain: this study leads to conclusions far different from the interpretations which has so far been suggested and offers a new approach to the task assigned to himself by the engraver. By engraving runes over the whole surface of the rock he gives birth to an unrivalled monument, the only monument worthy of his dead son's memory.

* Alain Marez est professeur honoraire de linguistique germanique et scandinave à l'université Michel de Montaigne - Bordeaux. 
Le monument suédois de Rök (Ög. 36), avec ses quelque 725 runes, constitue le texte le plus long du corpus runique sur pierre et son inscription se développe sur toute la surface disponible du support. Le texte ne pose pas de problèmes de déchiffrement, sauf sur sa partie inférieure, où les runes sont mutilées ; en revanche, son interprétation donne encore lieu à de nombreuses controverses. L'une d'entre elles concerne la formule qui rythme en une ample anaphore l'ensemble de l'inscription : <sakumukmini $>^{1}$; elle apparaît en effet sept fois (peut-être huit ?) sous des formes diverses, ce qui montre bien l'importance que lui accorde son auteur ${ }^{2}$. Son interprétation demeure malheureusement précaire et ambiguë, car les spécialistes se partagent en deux hypothèses fondées l'une et l'autre sur une découpe en mots erronée : <sakum + ukmini>, laquelle est obstinément reprise depuis qu'elle a été émise par Elias Wessén en $1958^{3}$. Une telle segmentation conduit à un contresens sur sa signification, car les deux hypothèses mettent en jeu des éléments improbables et négligent les évidences fournies par une analyse serrée de la séquence graphique dans son ensemble :

$<$ sakum $>=/$ sagum $/$ serait une $1^{\text {re }}$. P.pl. du prés. ind. ou subj. de /segja/ et l'expression d'un ton solennel de la poésie scaldique, ce qui est strictement invérifiable, « je dis (nous disons) »;

$<$ ukmini $>=/ \mathrm{u}(\mathrm{n})$ gmenni/ Dsg. « aux jeunes gens, à la jeunesse "; $<$ ukmini $>=<$ mukmini $>=$ /mugminni/ Asg. " la mémoire du peuple».

Deux interprétations seraient donc possibles, soit « je dis (je raconte) au jeunes gens », dans laquelle le groupe $<$ ukmini> a la fonction de $\mathrm{D}$, soit « je dis (je raconte) la mémoire populaire » où il apparaît comme un A. L'une comme l'autre sont peu convaincantes.

1 Les signes encadrés de $\langle\ldots\rangle$ notent les séquences graphiques, ceux bornés par [...] les chaînes phoniques et ceux délimités par /.../ les unités phonologiques correspondantes.

2 Sous sa forme complète

<sakumukminipat> - L'échange des deux butins / <sagwmogumeniPat> en partie en ancien fupark germanique ;

<sakumukminiuaim> en runes de substitution <airfbfrbnhnfinbantfnhnu> / <sakumukminipur> en runes à branches ;

1 en grande majorité (7/8 sur 11) en fupark germanique à 24 signes

1 en nouveau fupark à 16 signes (variante de Rök - kortkvistrunor ou runes suédo-norvégiennes)

2 en runes secrètes (substitution et runes à branches).

Sous sa forme abrégée

$<$ Patsakum annart $>$ - Introduction à la strophe sur Pjóðrikr / < Patsakumtualfta $>$ - Le cheval de Gunn ; $<$ Patsakumpritaunta $>$ - Les vingt rois dans l'île de Sjælland / <nukmini...>;

Toutes en nouveau fupark à 16 signes (variante de Rök)

3 Elias Wessén [1889-1981], "Runstenen vid Röks kyrka », Kungl.Vitterhets historie och Antikvitets Akademiens Handlingar, Filologisk-filosofiska serien, 5, article préfacé par son auteur en octobre 1957 et publié en 1958, 89 p.

Lis Jacobsen [1882-1961], « Rökstudier ", Arkiv för nordisk filologi, LXXVI, 1961, p. 1-50.

Ottar Grønvik, « Runeinnskriften på Rök-steinen », Maal og Minne, Hefte 3-4, 1983, p. 101-149. 
Cette séquence en nouveau fupark (variante de Rök) serait difficilement compréhensible si elle n'était pas réécrite, du moins partiellement, en runes germaniques (fupark à 24 signes). Désireux sans doute de faire étalage de sa science des runes, le graveur reproduit le message qu'il entend délivrer à ses lecteurs potentiels, créant ainsi un monument comparable à une pierre de Rosette du Nord! Seule une analyse rigoureuse et systématique de la séquence graphique en runes germaniques permet de reconstruire la chaîne phonétique sous-jacente.

Voici l'aspect des deux séquences graphiques, la première en runes germaniques, la seconde en fupark récent (variante de Rök ou runes à branches courtes).

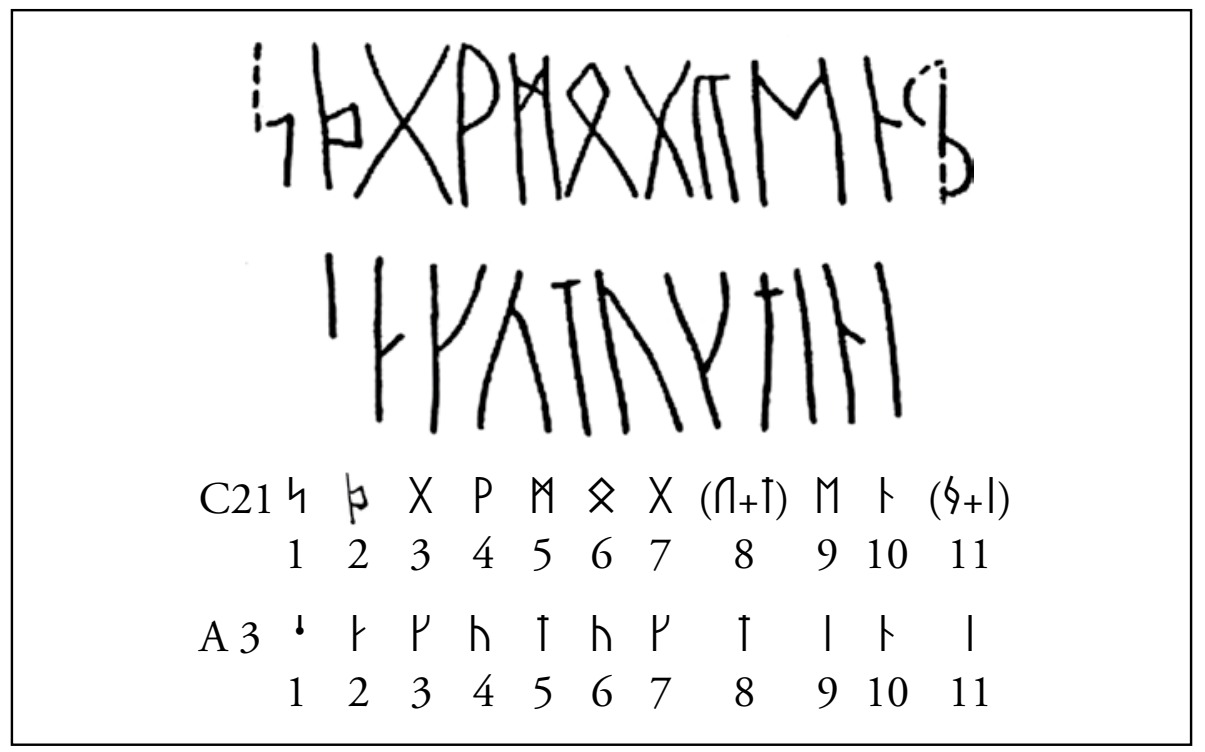

R2 : Le pictogramme de la hache $\boldsymbol{p}$ : le signe est unique dans toute la littérature runique et son caractère nettement figuratif (image d'une hache) interdit de le considérer comme une autre déformation de l'ancienne rune */jara/ dont toutes les formes sont non calibrées et non figuratives ( Il n'est pas douteux par ailleurs qu'il note [a] (a oral) non seulement dans la séquence qui nous occupe, mais aussi dans tout le reste de l'inscription où il apparaît à cinq reprises $(\mathrm{R} 13<\mathrm{pad}>=[\mathrm{pat}]$ " cela ", R17 <hoar $>=[\mathrm{hwaR}]$ " qui ? ", R26 - <ika > = [-inga] "-inga », R28 <uaRi > = [wæri] « aurait été ", R42 $<$ goąnaR $>=[\mathrm{kwanaR}]$ "d'une femme ». Ce dernier exemple montre d'ailleurs nettement que le graveur distingue entre /ą/ (a nasal) qu'il transcrit normalement par la rune* /ansuz/ « (dieu) Ase » et [a] (a oral) auquel il réserve la notation par le pictogramme. Une question demeure toutefois : pourquoi n'utilise-t-il pas pour noter [a] (a oral) les deux possibilités que lui offrent les inscriptions $d u$ 
Blekinge $\boldsymbol{*}$ et $\boldsymbol{\zeta}$ ? Les deux formes, apparues après la chute $\mathrm{du} / \mathrm{j}$ initial/ dans la rune */jara/ prennent naturellement la valeur de notation [a] (a oral) en vertu du principe acrophonique du nom des runes ${ }^{4}$ et sont considérées traditionnellement comme des "graphèmes de transition ", mais ne constituent pas une notation nette et tranchée entre $<\mathrm{a}>$ [a] (a oral) et $<a<$ [a]/ (a nasal) : outre le fait que $\mathbf{4}$ n’apparaît que sur Istaby avec la valeur exclusive de [a] (a oral), $\boldsymbol{*}$ entre en concurrence avec [a] (a nasal) en ancien fupark dans la transcription du même [a] (a oral) (cf. † Gummarp < st $\boldsymbol{* b} \boldsymbol{*}>$ alterne avec $<$ pri $\boldsymbol{\uparrow}>$ et Stentoften $<$ niuh $\boldsymbol{*}>$ alterne avec $<$ niuh $>$. On conçoit donc qu'une telle instabilité ne soit guère propice à l'adoption de l'un des deux graphèmes pour transcrire [a] (a oral). Si on ajoute à cette circonstance défavorable le fait que les inscriptions du Blekinge (650-750 ou 800) représentent des textes isolés assez distants dans le temps et dans l'espace de l'inscription de Rök, il n'est pas du tout invraisemblable que le graveur n'ait pas eu connaissance de leur existence. Enfin - et nous tenons sans doute là l'argument décisif en faveur de l'hypothèse du pictogramme -, la volonté de faire étalage de sa maîtrise dans l'usage des runes, son goût du secret et de la mystification trouvent leur réalisation idéale dans l'invention d'un nouveau signe graphique dont le nom respecte exactement le principe acrophonique : la hache se dit en effet /ax/f. (thème en /-iō/).

R4 P : C'est sous sa forme arrondie la rune 8 de la série germanique, laquelle porte le nom */wunjo/ « joie, plaisir ». Elle note à l'origine le glide bilabial /w/ et subsiste à l'initiale dans le groupe des inscriptions du Blekinge. Mais vers le début du IX ${ }^{e}$ siècle, à l'époque où le graveur réalise son œuvre, le signe devait avoir la valeur $/ \mathrm{u} /(* / \mathbf{u n j o} /)$ plus ou moins nasalisé et son choix n'est sans doute pas un hasard puisqu'il précède immédiatement la rune notant $/ \mathrm{m} /$ une autre nasale. Par ailleurs, le glide bilabial /w/ est toujours transcrit par la rune $\hat{\boldsymbol{X}}=\langle 0\rangle$ dans le reste de la séquence en runes germaniques, ce qui prouve bien que le lapicide ne connaît pas la valeur originelle du signe 5 .

$\mathrm{R} 4+\mathrm{R} 5:$ PM $=<\mathrm{u}+\mathrm{m}>$ : Wessén (1958) et Jacobsen (1961) ainsi que leurs successeurs considèrent l'association des deux runes comme la transcription d'un

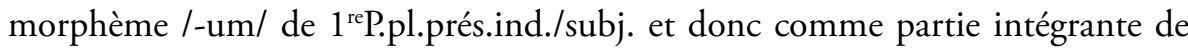
la forme verbale (segja). C'est là que se situe l'erreur funeste qui, pendant longtemps, a rendu l'interprétation de la formule précaire et ambiguë. L'absence de "ponctuation »-entendre par là de signes séparateurs entre les mots - est encore

4 Chaque rune du fupark a un nom : cf. $\langle\mathrm{f}>=$ fé « bétail, richesse », $\langle$ gebō $>$ " don, présent ", le son initial de ce nom indiquant la valeur phonétique (ou phonologique) du graphème, soit /f/spirante labiale sourde (fr. fête), /g/occlusive vélaire sonore (fr. galère).

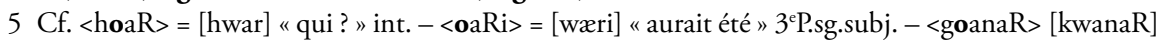
"d'une femme » Gsg.fém. 
coutumière sur les monuments à l'époque de Rök et cette ponctuation ne sera introduite de manière quasi systématique que lors de la période dite de Jelling pour les textes danois, c'est-à-dire dans la seconde moitié du IX siècle. Il convient donc de considérer $<u m>$ comme une séquence indépendante de la forme verbale

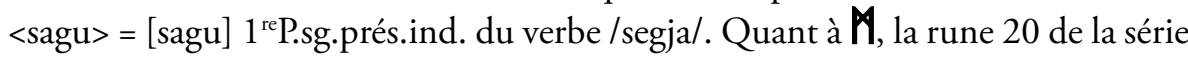
germanique */mannaz/ «homme » $<\mathrm{m}>[\mathrm{m}]$, elle note une occlusive labiale nasalisée prolongeant la chaîne phonique initiée par $/ \mathrm{u} / . \mathrm{PM}=\langle\mathrm{u}+\mathrm{m}\rangle=[\mathrm{um}]$. Mais, comme l'orthographe runique interdit absolument de réécrire deux fois le même caractère lorsque deux sons (ou phonèmes) semblables se succèdent immédiatement (règle de l'haplographie), [u] ne peut apparaître dans la séquence graphique. Il en va de même pour $\langle\mathrm{m}>$, dont l'haplographie avait déjà été pressentie par certains spécialistes puisque le mot suivant commence aussi par $<\mathrm{m}>$. La véritable chaîne phonique se présente donc ainsi /sagu (u)m (m...) /. On ne voit du reste pas comment le graveur aurait pu procéder pour la noter, puisqu'une séquence

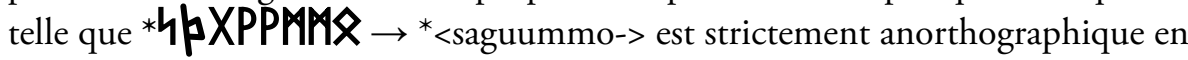
runique. Le résultat de cette double haplographie à la fois initiale et finale met paradoxalement en valeur l'élément essentiel de la chaîne phonique, c'est-à-dire la préposition [um].

$\mathrm{R} 8 \widehat{\mathrm{CT}}<\mathrm{u}+\mathrm{m}>$ : Il s'agit là d'une ligature ${ }^{6}$ et non d'un graphème simple, laquelle associe la rune $<\mathrm{u}>\mathrm{R} 2$ qui a la même forme en ancien et en nouveau fupark (et tolère une version inversée $\bigcap$ et $\Pi$ ) avec la rune $<\mathrm{m}>\mathrm{I}$ telle qu'elle apparaît dans le fupark de Rök! La forme du $<\mathrm{m}>$ ne fait aucun doute, même si le trait horizontal se trouve au sommet de l'axe vertical et ne le coupe pas, servant ainsi de lien ininterrompu au jambage de gauche qui ne fait d'ailleurs l'objet d'aucun commentaire puisque son existence n'est même pas mentionnée dans les études des runologues les plus connus! Il s'agit en fait de la rune $<\mathrm{u}>$ sous sa forme renversée, même si le trait n'est pas franchement courbe, mais plutôt parallèle à la haste voisine commune aux deux runes. La séquence se lit donc $<-\mathrm{g}(\mathbf{u})$ me $>$ et non <-gme> comme l'ont jusqu'à maintenant interprétée faussement les runologues.

R9 $M<$ < $>$ : La rune 19 dans l'inventaire de l'ancienne série germanique ; elle a pour nom */ehwaz/ " cheval " et note à l'origine une ouverture moyenne avant [e]. Au moment où le graveur compose son texte, elle a vraisemblablement pris la valeur de notation / j/ devant voyelle et /i/ devant consonne, cf. <iu>/iu/

6 Ligatures et haplographies procèdent du même principe d'économie de l'espace - particulièrement précieux en épigraphie et sur le monument de Rök gravé sur toutes ses faces !-, les premières en fusionnant deux (ou plusieurs) graphèmes en un seul nouveau, les secondes en n'écrivant qu'une seule fois un groupe de lettres redoublées : dans l'inscription de Rök, on relève 2 ligatures $<\mathrm{um}>$ et $<\mathrm{ij}>$ et 3 haplographies $<\mathrm{u}(\mathrm{u}) \ldots>,<\mathrm{m}(\mathrm{m}) \ldots>$ et $<\min (\mathrm{n}) \mathrm{i}>$. 
Asg. Roes (vers 800) et en nordique occidental (vieux norrois) <jør> désignation poétique du cheval " étalon ». Dans le contexte de l'inscription de Rök, le signe transcrit sans aucun doute /i/ en raison de l'élévation tonique due à la nasale redoublée qui suit.

$\mathrm{R} 10$ 卜 $<\mathrm{n}>$ : Le graphème occupe la huitième place dans l'inventaire du nouveau fupark (variante de Rök ou runes suédo-norvégiennes), la dixième dans celui des runes germaniques où il a une forme légèrement différente : le trait sécant traverse en biais la haste verticale $\$$. Le nom de la rune demeure le même : */naupiz/ "détresse, nécessité fatale " $<\mathrm{n}>=[-\mathrm{nn}-]$, car le redoublement d'une consonne ne trouve pas d'expression graphique comme l'exige l'orthographe runique (cas particulier d'haplographie).

R11 $\widehat{19}<\mathrm{i}+\mathrm{j}>$ : Il s'agit d'une nouvelle ligature associant la rune 12 de l'ancienne série germanique $\boldsymbol{S}<\mathrm{j}>* /$ jara/ « année fertile, prospère » et la rune $11 \mathrm{de}$ l'ancien ( 9 du nouveau fupark) $\mid<\mathrm{i}>* /$ isaz/ ou */isan/ " glace ». Elle ne peut que noter le son (phonème) /i/.

Un bilan de la séquence graphique (C21 dite en runes germaniques) ne laisse en réalité apparaître que 7 signes appartenant à l'ancien fupark, soit R1, R3, $\mathrm{R} 4, \mathrm{R} 5, \mathrm{R} 6, \mathrm{R} 7$ et $\mathrm{R} 9$, les autres se répartissant de la façon suivante : un pictogramme inconnu $\mathrm{R} 2 \boldsymbol{p}<\mathrm{a}>$ (a oral), une rune appartenant à la série du nouveau fupark (variante de Rök/ runes à branches courtes dite suédo-norvégiennes), R10 $\boldsymbol{R}<\mathrm{n}>=/ \mathrm{n} /$ et 2 ligatures R8 $\widehat{\Pi T}<\mathrm{um}>/$-um-/ et R11 $\overline{\mathbf{6 l}}<\mathrm{ji}>/ \mathrm{i} /$. L'utilisation du pictogramme se justifie comme nous l'avons vu et s'intègre parfaitement au système acrophonique du nom des runes. On ne voit pas en revanche pourquoi le lapicide emploie la rune $+<\mathrm{n}>=/ \mathrm{n} /$, forme nouvelle dans la variante de Rök (R10), alors qu'il avait à sa disposition celle de l'ancienne série germanique $\uparrow<\mathrm{n}>$ ! Quant aux deux ligatures, elles sont aisément explicables : elles mettent en jeu les runes $\bigcap<\mathrm{u}>$ et $\mathbf{I}<\mathrm{i}>$ qui ont toutes deux la même forme dans la série germanique et dans le nouveau fupark de Rök ; le graveur, fidèle à son dessein de rédiger la séquence en runes germaniques, c'est-à-dire incompréhensibles pour les lecteurs contemporains non initiés, doit donc à tout prix éviter de les employer. On a vu comment il contournait la difficulté pour la transcription de /-u/ devant nasale, mais dans le cas qui nous occupe, il a recours à un véritable subterfuge : en créant une association de deux signes appartenant à deux fupark différents, il parvient à donner l'illusion qu'il a recours à des runes de l'ancienne série germanique alors que les éléments en cause sont parfaitement identifiables individuellement $\widehat{\Pi \mathrm{T}}<\mathrm{u}+\mathrm{m}$ (nouveau fupark - variante de Rök) et $\overline{\mathbf{9}}<\mathrm{j}($ fupark germanique $+\mathrm{i}>$ ) . 
La séquence se présente donc sous la forme : < $\operatorname{sagwmog}(\mathrm{u})$ mini> et se laisse segmenter en mots de la façon suivante :

\section{lsagu-(u)m-(m)ågumi(n)nil}

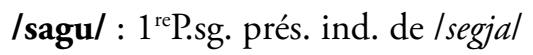

/-a-/ sous l'accent dans /sagu/ : Cette notation de la voyelle radicale s'explique sans doute par le fait que la palatalisation de /-a-/ par /j/ (métaphonie par /-i-, $-\mathrm{j}-/)$, ne s'est pas produite comme elle interviendra beaucoup plus tard en vieux norrois (nordique occidental) ; le vocalisme radical (sous l'accent) du groupe du présent est identique à celui du prétérit (sagða) et du P2 (sagðr) et la forme relève sans doute du nordique oriental.

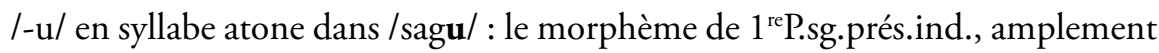
attesté pour les verbes apophoniques, ne l'est pas formellement pour les verbes faibles dans le corpus runique, bien que son existence ne fasse aucun doute pour les spécialistes ${ }^{7}$.

/(u) (m)/ : prép. + Acc. : "Tout autour de, sur toute la surface de » :

La préposition apparaît dans le corpus runique danois :

DR $-1 \underline{\text { Haddeby }} 1$ (pierre de) $:<$ pxtrekiaRxsatuxumxhaipabu $>-/ p a d r a n g i a R$ satu um Hepaby/

«Lorsque les jeunes guerriers firent le siège de Heðaby »

L'absence d'haplographie est due aux signes séparateurs en forme de $<\mathbf{x}>$ introduits assez tardivement en guise de ponctuation.

/(m)ogu-/ : nom. masc.sg. mögr m., thème en /-u-/ « fils, jeune homme » :

( $\mathrm{m}$ initial) élidé dans la graphie par haplographie obligée (règle de l'orthographe runique) : premier membre du mot composé /mogu-minni/.

$<-u>$ : voyelle thématique en fonction d'élément vocalique de liaison lorsque le nom (thème en /-u/) est le premier membre d'un mot composé. La persistance du formant thématique en cette position est une règle quasi générale concernant les thèmes en $/-\mathrm{u}-/^{8}$.

En ancien fupark, R6 <o $>$ transcrit le produit de la métaphonie de [a] par [u], soit une voyelle présentant les traits suivants : postérieure + , arrondie + , mi-ou-

7 Cf. Wolfgang Meid, Hans Krahe, Germanische Sprachwissenschaft, II, Formenlehre, Berlin, Walter de Gruyter, 1969, $\$ 85$, p. 120 et M. Syrett, The Unaccented Vowels of Proto-Norse, Odense, Nowele 11, $1994, \$ 8.1$, p. 237.

8 Ibid., $\$ 21$, p 20 et M. Syrett, op. cit., 4.4, p. 72. 
verte + (cf. sa notation en suédois moderne par le graphème $<$ å $>$ ). La rune $\hat{\boldsymbol{X}}<\mathbf{0}>$ disparait de l'inventaire des runes scandinaves et est remplacée par $\Lambda<\mathrm{u}>$.

Le terme apparaît sur les inscriptions danoises suivantes :

DR 685 - 357 Stentoften (post grandes invasions) - inscriptions du Blekinge (750-800) : Dsg. <(m)A(gi) u> - /magiu/ - « au fils »

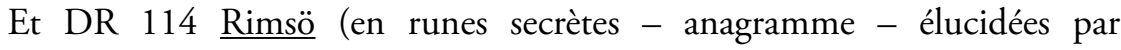
Wimmer) - période viking (Jelling) :

Dsg. <ikam:tsrau:mas:ipua $>/<[\ldots]$ aupi:sam:warst:maki $>$ Wá es d] $]$ áuði sem werst magi/ « la mort (de la mère) est le pire des malheurs pour un fils »

/- minni/ : nom neut. A.sg. ou pl., thème en /-ia-/ « monument funéraire, stèle runique (commémorative) »

DR 687 - 83 Sønder Vinge 2 - (per. Viking, post Jelling) :

Dsg. <ias :aupi:mini:pui> / as øpi minni pwi / « celui qui détruirait ce monument"

DR 94 Ålum 1 - (per. Viking, post Jelling)

Npl. < mini > Iminni / " ce monument (funéraire) »

Et DR 110 Virring - (Moyen Âge, fin du XII')

Apl. <k(a)rpi:m(i)n(i):pau> - /garpi minni pø/ - «fit ce monument"

Dans le corpus runique, il est parfaitement synonyme de /kum(b)ll ou de /sten $/{ }^{9}$ et on demeure surpris de le voir traduit par "mémoire " par des runologues aussi avertis que Wessén (1958) et Jacobsen (1961), lesquels commettent ce faisant un anachronisme patent en donnant au terme le sens qu'il a en suédois contemporain. Mais l'emploi du mot composé entier est attesté de façon remarquable dans des circonstances en tout point semblables à celles qui président à la rédaction de la pierre de Rök - un père grave un monument dédié à son fils défunt -, sur l'inscription de Kjølevik (Rogaland, Norvège) quelque trois siècles auparavant $(\text { vers } 450)^{10}$ :

$<$ hlaaiwidomaguminino> /hlai(wa) aiwid maguminni(i)no/ " j'ai fait le monument (funéraire) de mon fils "

Une comparaison des deux séquences graphiques, celle de la série germanique et celle rédigée en fupark récent (variante de Rök (kortkvistrunor) ou runes suédonorvégiennes) montre à l'évidence une correspondance parfaite à une exception

9 À l'exception de U114 (Suède), où le terme a nettement le sens de « souvenir, mémoire » :<(p)it skal at minum mana mipan min li[fa $]>$.

10 Cf. : Alain Marez, Die Kjolevik-Inschrift (Rogaland, Norwegen), eine neue kritische Deutung in Zeitschrift für deutsche Philologie, Bd.120, Heft 3, 2001, p.413-420. 
près : la séquence en nouveau fupark a éliminé le $<-\mathrm{u}->=/$-u-/ qui faisait office de voyelle de liaison dans le composé $<\operatorname{mog}-\mathbf{u}-$ meni> ; sa disparition n'a rien d'étonnant après la grande crise épigraphique du VIII siècle due à l'évolution de la chaîne phonique, soit :

$$
\begin{gathered}
<\text { sagwmog }(\mathrm{u}) \mathrm{meni}>=\text { /sagu }(\mathrm{u}) \mathrm{m}(\mathrm{m}) \text { åg }(\mathrm{u}) \text { minni/ } \\
<\text { sakumukmini }>
\end{gathered}
$$

Au terme d'une analyse épigraphique méthodique et raisonnée, on observe donc que les interprétations proposées jusqu'à maintenant sont insoutenables, car elles résultent à la fois d'une découpe erronée de la séquence graphique assortie de lourdes négligences dans l'analyse des graphèmes runiques. La signification véritable de la formule est bien :

\section{/sagu um mågminni/}

" Je dis tout autour (c'est-à-dire : sur toute la surface) du monument (funéraire) de mon fils »

Le lapicide, en l'occurrence Varinn le père de Vemoð, informe le(s) lecteur(s) éventuel(s) de son projet : réaliser en hommage à son fils défunt un monument funéraire original entre tous. Aucune stèle runique n'égalera celle qu'il est en train de réaliser, car aucune ne sera inscrite sur toute sa surface comme l'est la pierre qu'il entreprend de graver! Rien à voir par conséquent avec un projet "pédagogique "à l'adresse de la jeunesse ni avec une fonction de "récitant " de légendes engrangées dans la "mémoire du peuple ", mais l'expression d'une fierté certaine pour son œuvre affirmée tout au long de l'inscription dans trois registres : l'un, parfaitement lisible par les contemporains, celui rédigé en runes récentes (variante de Rök), le second en runes de l'ancien fupark germanique pour les lecteurs érudits, le troisième enfin en runes secrètes réservé aux seuls initiés. 